Noname manuscript No.

(will be inserted by the editor)

\title{
Exclusive Scattering from Unpolarized and Polarized Deuteron
}

\author{
Sabine Jeschonnek • J. W. Van Orden
}

Received: date / Accepted: date

\begin{abstract}
We present results for exclusive electron scattering from polarized and unpolarized deuteron. We employ the Gross equation to describe the deuteron ground state, and we use the SAID parametrization of the full NN scattering amplitude to describe the final state interactions (FSIs). We include both on-shell and positiveenergy off-shell contributions in our FSI calculation. We discuss properties of various asymmetries accessible with a polarized deuteron target and/or a polarized beam.
\end{abstract}

PACS 25.30.Fj, 21.45.Bc, 24.10.Jv

\section{Introduction}

Currently, there are many exclusive electron scattering experiments with light nuclei as targets at Jefferson Lab. These experiments have a variety of goals: finding out about the nuclear ground state and its high momentum components, the reaction mechanism, measuring the neutron form factors, and searching for color transparency.

Electron scattering from a nucleus, in the most naive plane wave impulse approximation (PWIA) picture, would have a cross section proportional to the spectral function of the nucleus, i.e. it would yield information about the momentum distribution of

Relativistic Description of Two- and Three-Body Systems in Nuclear Physics, ECT*, October 19-13 2009

Notice: Authored by Jefferson Science Associates, LLC under U.S. DOE Contract No. DEAC05-06OR23177. The U.S. Government retains a non-exclusive, paid-up, irrevocable, worldwide license to publish or reproduce this manuscript for U.S. Government purposes.

Sabine Jeschonnek

The Ohio State University, Physics Department, Lima, OH 45804

Tel.: +419-995-8201

Fax: +419-995 8491

E-mail: jeschonnek.1@osu.edu

J. W. Van Orden

Department of Physics, Old Dominion University, Norfolk, VA 23529 and

Jefferson Lab, 12000 Jefferson Avenue, Newport News, VA 23606

E-mail: vanorden@jlab.org 

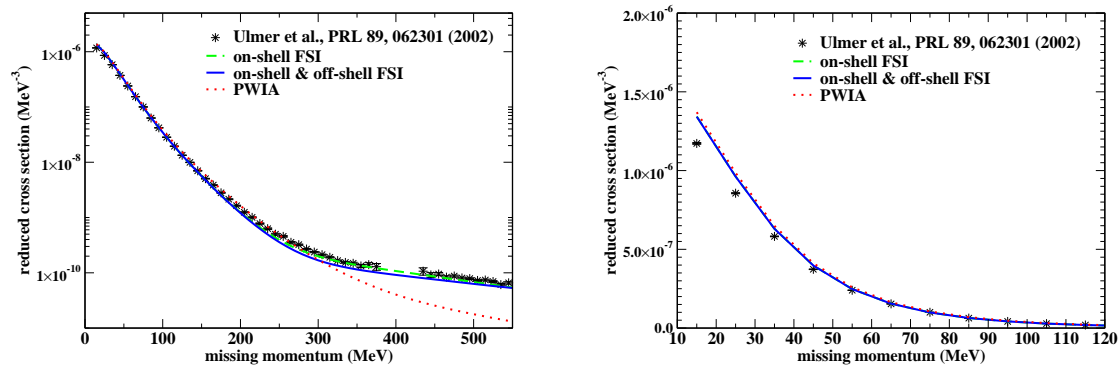

Fig. 1 Reduced cross section calculated for the kinematics of Ref.[3], together with the data from [3]. The right panel shows the same results on a linear scale, for lower missing momenta.

the nucleons in the target right away. In reality, the reaction mechanism is much more complicated, and in particular final state interactions (FSIs) between the two outgoing nucleons have to be taken into account. FSIs are expected to be the dominant part of the reaction mechanism at $\mathrm{GeV}$ energies, i.e. for experiments performed at Jefferson Lab. Below, we will briefly discuss how we implement FSIs. This method works fine for any value of the momentum transfer squared, $Q^{2}$, even for values of $Q^{2}$ of less than 1 $\mathrm{GeV}^{2}$. However, in these situations, meson exchange currents and isobar contributions will play a role. Currently, we do not include these effects in our calculations.

The main features of our calculation are that we use a relativistic ground state wave function, a positive-energy current operator, and a description of the FSIs that includes all spin-dependent terms of the nucleon - nucleon scattering amplitude. For details of the formalism, see [1].

The main difference to many other high quality calculations using the generalized eikonal approximation [?,?,?] or a diagrammatic approach [?] is the inclusion of all the spin-dependent pieces in the nucleon-nucleon amplitude. Full FSIs have recently been included in [?].

\section{Unpolarized Deuteron Targets}

Currently, $D\left(e, e^{\prime} p\right)$ data from Jefferson Lab's Hall A taken at $Q^{2}$ of $3.5 \mathrm{GeV}^{2}$ and $2.1 \mathrm{GeV}^{2}$ are analyzed [2]. Data taken at a lower $Q^{2}$ have been published already [3]. The kinematics employed there were a beam energy of $3.1095 \mathrm{GeV}, Q^{2}=0.665 \mathrm{GeV}^{2}$, $x_{B j}=0.964$, and $\phi_{p}=180^{\circ}$. The data and our results are shown in Fig. 1 . In the left panel, we show all the data taken. At medium and large missing momenta, it is obvious that the PWIA calculation fails miserably, as expected, and that the inclusion of FSIs brings the calculations much closer to the data. At a lower four-momentum transfer, one expects meson exchange currents to play a role at medium missing momenta, and we attribute the small remaining discrepancy between our curves and the data to the lack of meson exchange currents. The most interesting region, however, is the low missing momentum region. For a long time, a systematic deviation between theory and experiment was observed for exclusive electron scattering from the deuteron at low missing momenta, see [4]. We show our low missing momentum results in the right panel, and observe an improvement compared to the calculations reported in [3]. It is 


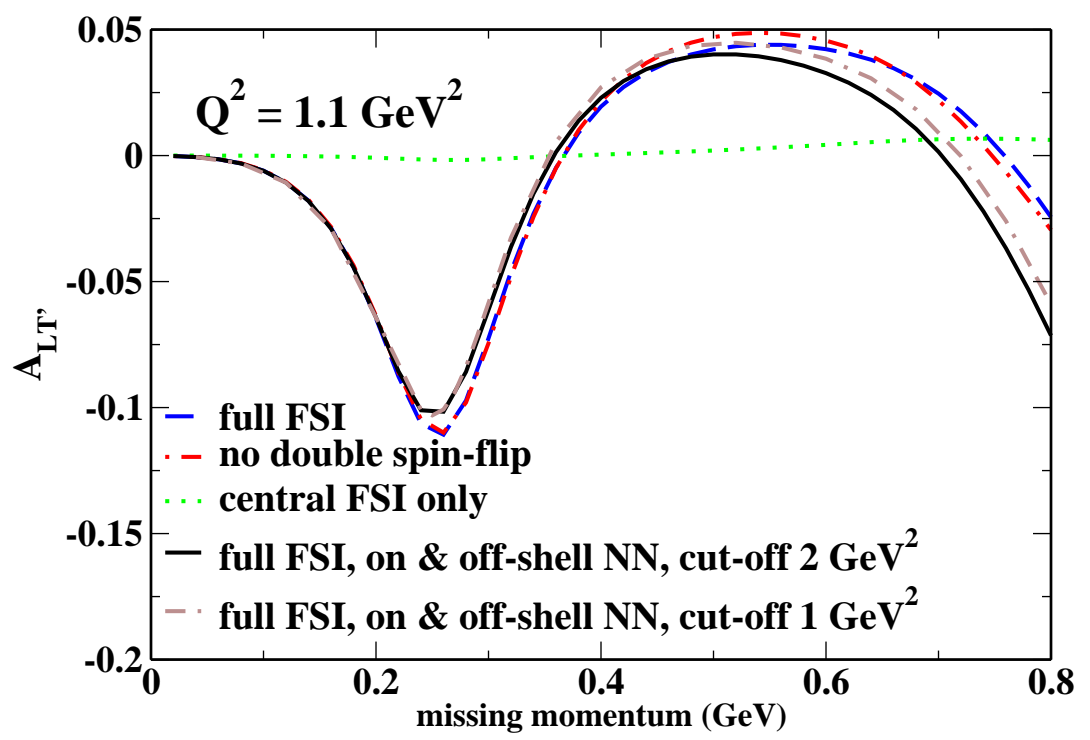

Fig. 2 The $L T^{\prime}$ asymmetry calculated for $Q^{2}=1.1 \mathrm{GeV}^{2}$, a beam energy of $2.6 \mathrm{GeV}$, and $x_{B j}=0.976$.

clear from the figure that the FSIs (both on-shell and off-shell) represent only a small correction, and that almost all the strength stems from the PWIA. While we do not agree completely with the data at the very lowest missing momenta, our results are an improvement. The main difference between present calculation and the calculations reported in [3] is that we use a relativistic ground state wave function.

In our formalism for the FSI, we include all parts of the nucleon-nucleon scattering amplitude. It is interesting to note that even for calculating the unpolarized cross section, the spin-dependent pieces of the $p n$ amplitude contribute significantly in the diffractive peak. When considering spin observables, these effects become even more important. As an example, we show results for the $L T^{\prime}$ asymmetry, which is closely related to the fifth response function.

$$
A_{L T^{\prime}}=\frac{v_{L T^{\prime}} R_{L T^{\prime}}}{v_{L} R_{L}+v_{T} R_{T}+v_{T T} R_{T T}}
$$

It vanishes in PWIA, and it has recently been measured at Jefferson Lab's Hall B. In Fig. 2, we show our results for FSI with only the central, spin-independent term, for FSI with the central and spin-orbit term, and for the full FSI, including also the three double spin-flip terms. It is clear that the central FSI alone is not sufficient - it leads only to a small deviation from zero. Once spin-dependence is included in the FSI, a dip at lower missing momentum, a change of sign, a bump at medium missing momentum, and finally another change of sign emerge. The chosen kinematics are similar to the kinematics of the Jefferson Lab experiment. Due to $x_{B j} \approx 1$, we do not expect a large 

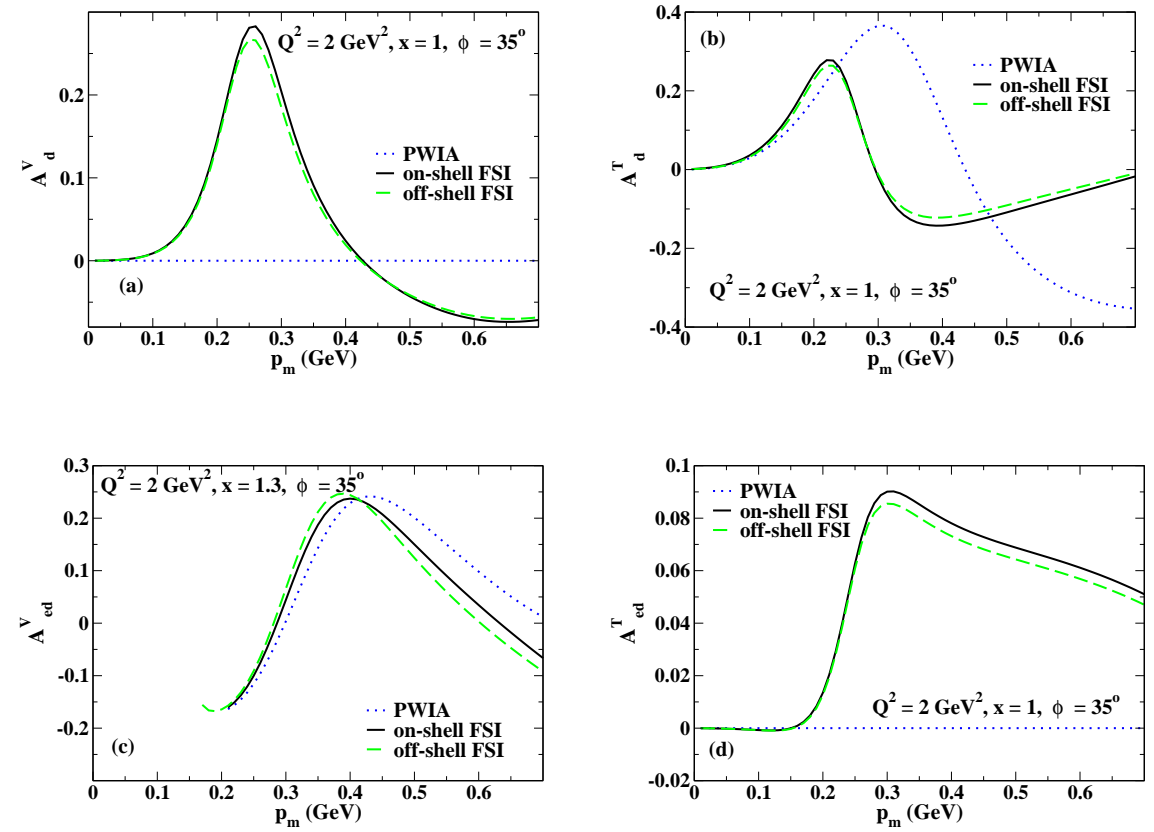

Fig. 3 The four asymmetries accessible with polarized target calculated for $Q^{2}=2 \mathrm{GeV}^{2}$, a beam energy of $5.5 \mathrm{GeV}, x_{B j}=1$, and $\Phi=35^{\circ}$.

contribution from the off-shell FSIs, and this is confirmed by the calculation. Even choosing a considerably larger cut-off of $2 \mathrm{GeV}$ leads only to a very minor correction at larger missing momenta. Our results do a good job describing the preliminary data from Jefferson Lab [5].

\section{Polarized Deuteron Targets}

Deuteron targets have spin 1 and may be vector-polarized and tensor polarized. The axis used as polarization axis is different from one experiment to the other, we use a polarization axis along the beam direction in our numerical results. In order to allow for arbitrary polarizations and polarization axes, we use a density matrix formalism and a rotation. For details, see [6].

The single and double asymmetries for vector and tensor polarizations are defined as

$$
\begin{aligned}
A_{d}^{V} & =\frac{v_{L} R_{L}\left(\widetilde{T}_{10}\right)+v_{T} R_{T}\left(\widetilde{T}_{10}\right)+v_{T T} R_{T T}\left(\widetilde{T}_{10}\right)+v_{L T} R_{L T}\left(\widetilde{T}_{10}\right)}{\widetilde{T}_{10} \Sigma} \\
A_{d}^{T} & =\frac{v_{L} R_{L}\left(\widetilde{T}_{20}\right)+v_{T} R_{T}\left(\widetilde{T}_{20}\right)+v_{T T} R_{T T}\left(\widetilde{T}_{20}\right)+v_{L T} R_{L T}\left(\widetilde{T}_{20}\right)}{\widetilde{T}_{20} \Sigma} \\
A_{e d}^{V} & =\frac{v_{L T^{\prime}} R_{L T^{\prime}}\left(\widetilde{T}_{10}\right)+v_{T^{\prime}} R_{T^{\prime}}\left(\widetilde{T}_{10}\right)}{\widetilde{T}_{10} \Sigma}
\end{aligned}
$$



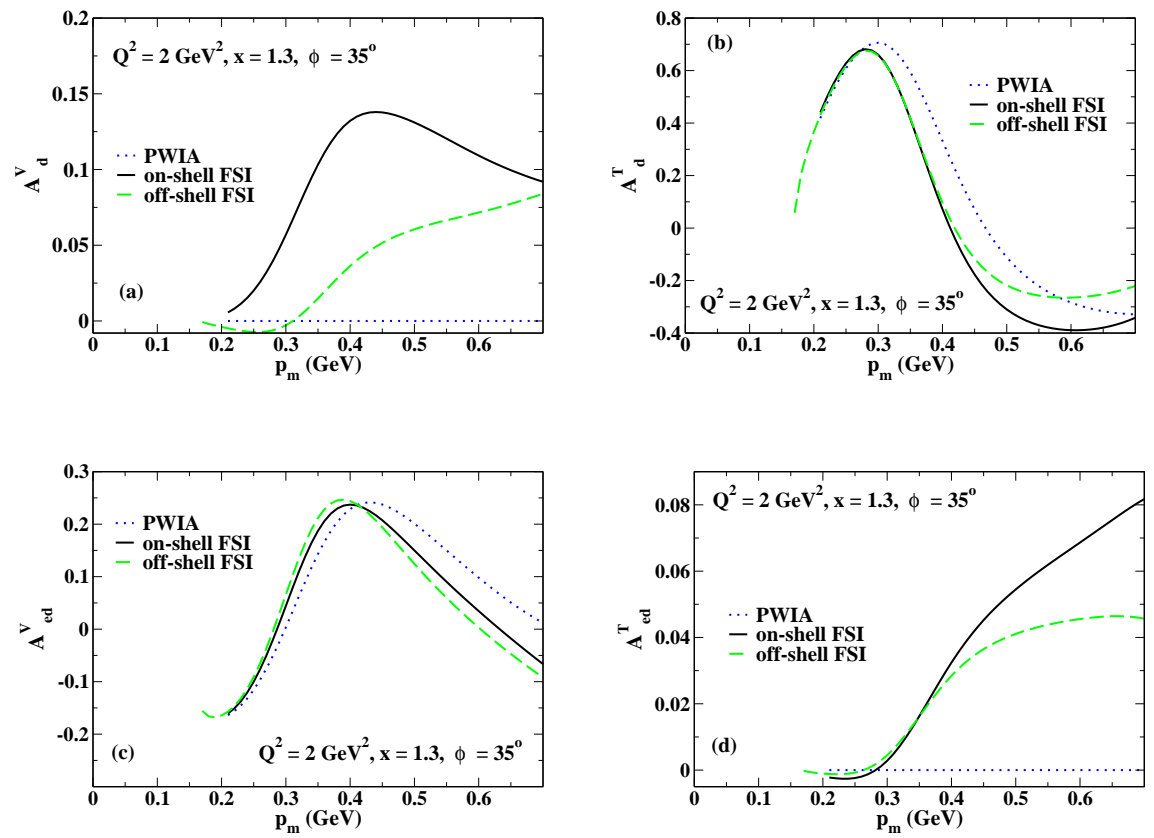

Fig. 4 The four asymmetries accessible with polarized target calculated for $Q^{2}=2 \mathrm{GeV}^{2}$, a beam energy of $5.5 \mathrm{GeV}, x_{B j}=1.3$, and $\Phi=35^{\circ}$.

$$
A_{e d}^{T}=\frac{v_{L T^{\prime}} R_{L T^{\prime}}\left(\widetilde{T}_{20}\right)+v_{T^{\prime}} R_{T^{\prime}}\left(\widetilde{T}_{20}\right)}{\widetilde{T}_{20} \Sigma}
$$

where

$$
\Sigma=v_{L} R_{L}(U)+v_{T} R_{T}(U)+v_{T T} R_{T T}(U)+v_{L T} R_{L T}(U) .
$$

All four asymmetries are shown for quasi-free kinematics, i.e. $x_{B j}=1$, in Fig. 3 . Two asymmetries, $A_{d}^{V}$ and $A_{e d}^{T}$, are zero in PWIA. FSIs are important for all asymmetries, and there is not much influence from the off-shell FSI, as expected at $x_{B j}=1$. Fig. 4 shows the asymmetries in the same kinematics, but for $x_{B j}=1.3$. Now, off-shell FSI effects are significant for $A_{d}^{V}$ and $A_{e d}^{T}$, and visible for higher missing momenta for the two other asymmetries. Naturally, off-shell FSIs are more important for the asymmetries that vanish in PWIA. It has been shown [6], using parity and time reversal arguments, that $A_{d}^{V}$ and $A_{e d}^{T}$ must both vanish in PWIA. They also share the same symmetry properties with respect to $\Phi_{p}$, the azimuthal angle of the outgoing proton. As demonstrated in the two previous figures, they also react similarly to FSIs. Our results suggest that very similar information might be gleaned from measuring either of these asymmetries. This is of practical importance as experiments on vector polarized targets are much easier to perform at JLab.

In Fig. 5, we show the contributions of the different parts of the proton-neutron scattering amplitude to the asymmetries. Central FSIs only do not describe $A^{V} d$ and $A_{e d}^{T}$ at all, but do give a rough picture of the other two asymmetries. Once single spin-flip terms are included, the shapes are reproduced for all asymmetries, but the 

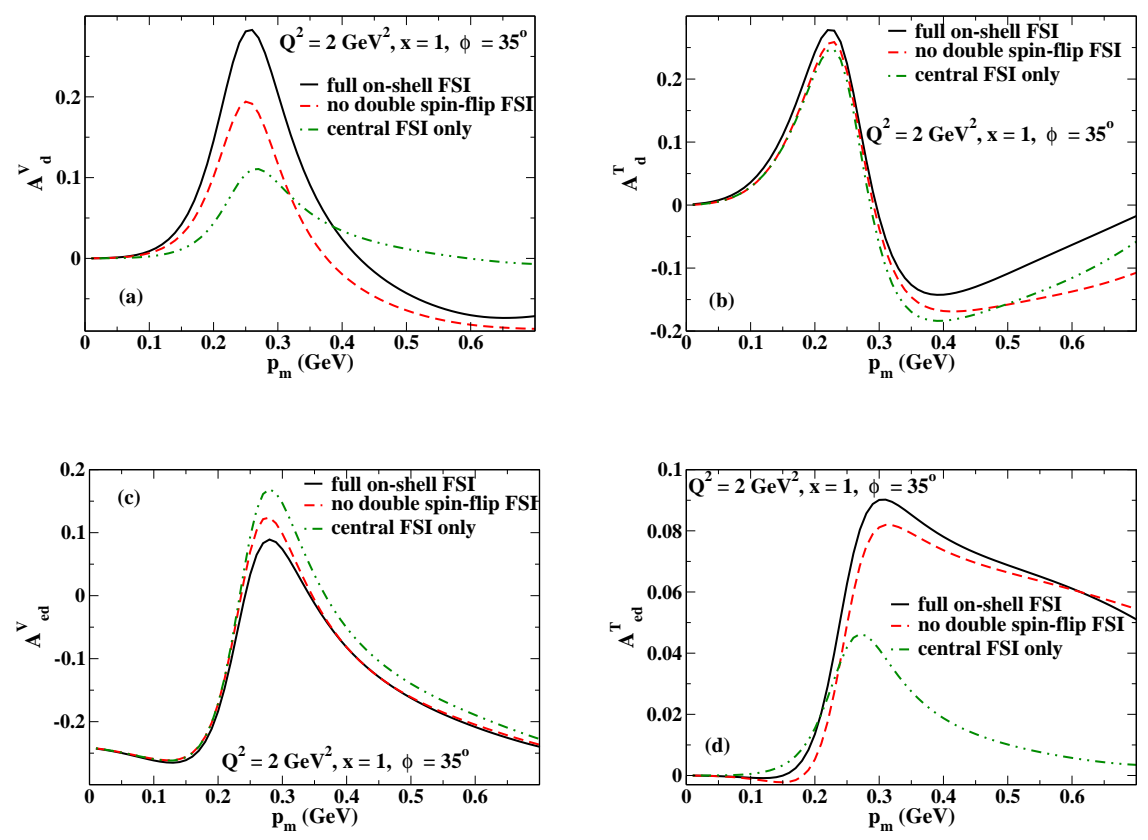

Fig. 5 The four asymmetries accessible with polarized target calculated for $Q^{2}=2 \mathrm{GeV}^{2}$, a beam energy of $5.5 \mathrm{GeV}, x_{B j}=1$, and $\Phi=35^{\circ}$. The contributions of the different parts of the FSIs are shown.

magnitude still receives considerable corrections from the double spin-flip terms, especially for $A_{d}^{V}$.

\section{Outlook}

Acknowledgements This work was supported in part by funds provided by the U.S. Department of Energy (DOE) under cooperative research agreement under No. DE-AC05-84ER40150 and by the National Science Foundation under grant No. PHY-0653312.

\section{References}

1. S. Jeschonnek and J. W. Van Orden, Phys. Rev. C 78, 014007 (2008).

2. Jefferson Lab Experiment E01 - 020, spokespersons W. Boeglin, M. Jones, A. Klein, P. Ulmer, J. Mitchell, E. Voutier.

3. P. E. Ulmer et al., "H-2(e,e' p)n reaction at high recoil momenta," Phys. Rev. Lett. 89, 062301 (2002).

4. Werner Boeglin, talk at the 2005 Workshop on "Probing microscopic structure of the lightest nuclei in electron scattering at JLab energies and beyond", Trento, Italy July 25-30, 2005, http://www.fiu.edu/ sargsian/ect05

5. G. Gilfoyle, spokesperson, Jefferson Lab Hall B, E5 run period; G.P. Gilfoyle, (the CLAS Collaboration), 'Out-of-Plane Measurements of the Fifth Structure Function of the Deuteron', Bull. Am. Phys. Soc., Fall DNP Meeting, DF.00010(2006). 
6. S. Jeschonnek and J. W. Van Orden, "Target Polarization for ${ }^{2} \mathbf{H}\left(e, e^{\prime} p\right) n$ at $\mathrm{GeV}$ energies," Phys. Rev. C 80, 054001 (2009).

7. S. Jeschonnek and J. W. Van Orden, "Ejectile Polarization for ${ }^{2} H\left(e, e^{\prime} \mathbf{p}\right) n$ at GeV energies," Phys. Rev. C 81, 014008 (2010). 